Revue d'histoire de l'Amérique française

REVUE D.HISTOIRE DE L'AMÉRIQUE FRANÇAISE

\title{
La cartographie de la région du lac Mistassini
}

Essai bibliographique

\section{Jacques Rousseau}

Volume 3, numéro 2, septembre 1949

URI : https://id.erudit.org/iderudit/801572ar

DOI : https://doi.org/10.7202/801572ar

Aller au sommaire du numéro

Éditeur(s)

Institut d'histoire de l'Amérique française

\section{ISSN}

0035-2357 (imprimé)

1492-1383 (numérique)

Découvrir la revue

\section{Citer ce document}

Rousseau, J. (1949). La cartographie de la région du lac Mistassini : essai bibliographique. Revue d'histoire de l'Amérique française, 3(2), 289-312. https://doi.org/10.7202/801572ar d'utilisation que vous pouvez consulter en ligne.

https://apropos.erudit.org/fr/usagers/politique-dutilisation/ 


\section{BIBLIOGRAPHIE}

\section{LA GARTOGRAPHIE DE LA REGION DU LAC MISTASSINI}

\section{ESSAI BIBLIOGRAPHIQUE}

\section{INTRODUCTION}

Le lac Mistassini est l'un des plus grands du Canada, après les Grands Lacs. Situé à mi-chemin entre le lac Saint-Jean et la baie James, il mesure un peu plus de cent milles de long et se déverse dans la baie James par la rivière Rupert. Pour atteindre la "mer du nord" par terre, c'était l'une des voies normales pour les voyageurs de la Nouvelle-France ${ }^{1}$.

Sous le régime français, Guillaume Couture s'y rendit en 1663. Il fut suivi par le père Charles Albanel, en 1672, le père François de Crespieul en 1674, Louis Jolliet en 1679. Vers 1700, Dorval y organise la traite des fourrures. En 1792, le botaniste français André Michaux y fait la première exploration botanique. En 1870, James Richardson, suivi l'année suivante par Walter McOuat, en commence l'exploration géologique. John Galbraith y passe en 1881. Puis en 1884 et 1885 , John Bignell et A.P. Low entreprennent d'en tracer la première carte complète ${ }^{2}$. L'arpenteur C.-E. Lemoine s'y rend en 1899 pour en dresser une nouvelle carte; puis, l'arpenteur Henri Bélanger dirige en 1929 la préparation du premier plan complet de cette grande nappe d'eau.

1. Jacques Rousseau, "Le voyage d'André Michaux au lac Mistassini en 1792". Reoue d'Histoire de l'A mérique française, 2(déc. 1948): 390-423. Aussi tirage d̀ part, avec changement de pagination et quelques corrections, Memoires du Jardin botanique de Montréal, No 3,1948, 34 p. Cetto étude renferme des notes historiques et bibliographiques sur les anciens voyages au lac Mistassini.

2. Jacques Rousseau, "Bataille de sextants autour du lac Mistassini". L'Action universitaire (Montréal), 14 année (No 2). 99-116. (Janvier 1948), Etude critique du conflit Bignell-Low. 
En 1938, G.W.H. Norman y fait des études géologiques. En 1943, le père Arthème Dutilly et l'abbé Ernest Lepage, après avoir remonté la Rupert, consacrent quelques jours à l'exploration botanique de ce lac. Ces brèves mentions ne prétendent pas épuiser, loin de là, la liste des voyageurs qui se sont rendus au lac Mistassini. Elles donnent au moins un aperçu des princjpales étapes de l'exploration du lac avant 1943.

De 1944 à 1948, j'ai fait cinq séjours au lac Mistassini, accompagné chaque fois par l'une des personnes suivantes: Ernest Rouleau, de l'Institut botanique de l'Université de Montréal, Jean-Paul Cuerrier, de l'Institut de Biologie de l'Université de Montréal, MadeleineA. Rousseau, Jean-Paul Vinay, professeur de phonétique à la faculté des lettres de l'Université de Montréal. En janvier 1948, toutefois, je m'y rendis seul pour vivre avec des Indiens sous la tente. Le premier objet de ces voyages était une exploration botanique aussi complète que possible de la région; mais les aspects ethnologiques, zoologiques et géographiques firent également l'objet d'observations assez pouヶsées.

La présente étude n'est qu'une section d'une bibliographie d'environ 500 titres, touchant à tous les aspects intéressant la région et comprenant les chapitres suivants: 1. Histoire, 2. Explorations gégraphiques et géologiques, 3. Botanique, 4. Zoologie, 5. Ethnologie, 6. Linguistique, 7. Météorologie, 8. Littérature, 9. Cartographie ancienne et moderne, 10. Manuscrits, 11. Travaux en cours. Cette compilation, encore manuscrite et où chaque article est l'objet de commentaires, couvre non seulement les travaux les plus importants, mais aussi les moindres entrefilets de journaux relatifs à la région. L'expérience m'a appris en effet que le moindre articulet anonyme d'un journal peut souvent fournir d'utiles précisions.

La portée de cette étude dépasse réellement le titre que je lui ai donné. Pour toutes fins pratiques, c'est presque la bibliographie de la zone subarctique du Québec.

Ce travail sur la cartographie n'a pu être mené à point sans la collaboration de plusieurs personnes. Je tiens a mentionner particulièrement: le R.P. Jean Delanglez s.j., le chanoine Victor Tremblay, de Chicoutimi, MM. Georges Côté et Isaie Nantais, du service des arpentages de Québec; MM. Gustave Lanctot, Séraphin Marion et A.J.H. Richardson, des Archives canadiennes, Ottawa; M. Léopold Desrosiers, Mlles Germaine Laflamme et Florina Brosseau, de la 
Bibliothèque municipale de Montréal; Mme Béatrice Grenier Bergeron.

Lorsque les dimensions des cartes sont indiquées, la largeur est avant la hauteur.

Jacques Roussenu

Jardin Botanique de Montreal

\section{A. Ouvrages de réferences}

1. Crouse, Nellis M. Contributions of the Canadian Jesuits to the geographical knouledge of New France, 1632-1675. A thesis presented to the faculty of the graduate school of Cornell University in partial fulfillment of the requirements for the degree of doctor of philosophy. $175 \mathrm{p} .14$ reproductions de cartes anciennes, (Cornell publications printing Co) Ithaca, N.Y., June, 1924.

2. [ HARRISSE, H. ]. Notes pour servir à l'histoire, à la bibliographie et à la cartographie de la Nouvelle-France et des pays adjacents, 1547-1700. 367 p., (Tross), Paris, 1872.

Renferme des commentaires sur les cartes de Jolliet.

3. MArcel, Gabriel. "Cartographie de la Nouvelle-France." Supplement à l'ouvrage de M. Harrisse. Publie avec des documents inédits. (Extrait de la Revue de Géographie), 41 p., Paris, 1885.

Renferme notamment la description des cartes suivantes: No 15. Carte de Louis Jolliet, 1684 (citée plus loin). Aussi texte d'une lettre de Jolliet accompagnant l'envoi d'une autre carte, ne couvrant pas la région du lac Mistassini. - No 69. Carte en couleurs du père Laure. (Sans doute la carte reproduite par La Rochemonteix et citée plus loin).

4. Roy, J.-Edmond. "Rapport sur les archives de France relatives à l'histoire du Canada." Publications des Archives du Canada, No 6, 1093 p., Ottawa, 1911.

5. VooRHIs, Ernest. Historic forts and trading posts of the French regime and of the English fur trading companies. 1930.

6. [ WhITE, James]. Forts and trading posts in Labrador peninsula and adjoining territory. In the Privy council: in the matter of the boundary between the Dominion of Canada and the colony of Newfoundland in the Labrador peninsula. 67 p. et une carte, citée plus loin. (F.A. Acland, Printer to the King's most excellent majesty), Ottawa, 1926.

Ce mémoire, sans nom d'auteur, et qui devait faire partie du factum préliminaire, ne semble pas inclus dans le grand factum en 12 volumes, publié à Londres en 1926-1927.

\section{B. Cartographie ancienne}

Les cartes sont citées dans l'ordre chronologique. Sont considérées comme anciennes, pour les fins de cette bibliographie, les cartes anté- 
rieures à l'expédition Bignell-Low, 1884-1885. Les cartes consultées font partie des collections des Archives canadiennes, Ottawa, de la bibliothèque municipale de Montréal (Collection Gagnon), de la Société historique du Saguenay, de Jacques Rousseau, de M. Fritz Knechtel, Hanover, Ont. Les cartes citées de cette dernière collection avaient été rassemblées pour la plupart par Voorhis lors de la préparation de son étude citée plus haut. Monsieur Knechtel a donné plusieurs de ces cartes à la Société historique du Saguenay. Aussi la mention "collection Knechtel" peut aussi bien englober des cartes appartenant maintenant à la Société historique du Saguenay. Comme les collections de cartes anciennes sont assez rares chez nous, il a paru utile d'indiquer dans chaque cas l'endroit où fut consultée la carte décrite. Quelques cartes sans date de publication, ont été datées approximativement. Ce sont les Nos 20, 21, 24, 34, 41, 42, 44, 45 et 50. Je n'ai pu dater, même approximativement, la carte 68 .

7. Champlain, Samuel de, 1632. "Carte de la nouuelle france, augmenteo depuis la / derniere, seruant a la nauigation faicte en son vray / Meridien, par le Sr de Champlain Capitaine pour le Roy / en la Marine; lequel depuis l'an 1603 jusques en l'année / 1629; a descouuert plusieurs costes, terres, lacs, riuieres, / et Nations de sauuages, par cy deuant incognuës comme / il se voit en ses relations quil a faict Imprimer en 1632. / ou il se uoit cette marque [ marque représentant un petit drapeau divisé en quadrants par une croix ] ce sont habitations / qu'on faict les François." Dimensions (d'après l'édition de la Champlain Society): $86 \mathrm{~cm}$ x $53 \mathrm{~cm}$.

Grande carte hors texte, accompagnant les œuvres de Champlain (dans un portefeuille, dans l'édition de la Champlain Society). Cette carte ne men tionne pas nommément le lac Mistassini, mais il est possible que ce soit l'un de ces lacs que Champlain a représenté à l'est de la baie James.

8. Borsseau, Jean. 1643. Description de la / Novvelle France : ou sont remarquées les diuerses habitations / des Francois, despuis la premiere descouuerte / jusques a present, receuillie et dressée sur / diuerses relations modernes, / 1643 / A Paris, chez Jean Boisseau, Enlumineur du / Roy pour les Cartes Geographiques, en / l'Isle du Palais à la Royalle / Fontaine de Jouuence." Dimensions de l'original: $55 \mathrm{~cm}$. large $35 \mathrm{~cm}$. haut.

Archives canadiennes, Ottawa. Le lac Mistassini n'est pas nommément représenté, mais ce pourrait être l'un des lacs au nord ou au nord-ouest du lac St-Jean. Cette carte s'inspire de celle de Champlain. Carte sans valeur (Delanglez in literis).

9. Sanson d'Abbeville, N., 1650. "Amerique / Septentrionale / Par N. Sanson d'Abbeville Geog. du Roy / A Paris / Chez l'Auteur / Et chez Pierre Mariette rue S. Iacques à l'Esperance / 1650 / Auec priuilege du Roy pour vingt Ans." 
Apparentée à la carte de Champlain, du moins pour le territoire de Mistassini, où les éléments sont à peu près les mêmes. Carte reproduite (hors texte) dans Crouse, Nellis M., Contributions of the Canadian Jesuits to the geographical knowledge of New France, 1632-1675. (Pour référence, voir Crouse, 1).

10. Sanson d'Abbeville, N., 1656. "Le Canada, ou / Nouvelle France \&c. / Ce qui est le plus advance vers le Septentrion / est tiré de diverses Relar tions des Anglois, Danois, Sc. / Vers le Midy les Costes de Virginie, Nouvlle Suede, / Nouveau Pays Bas, et Nouvelle Angleterre / Sont tirées de celles des Anglois, Hollandois, \&c. / La Grande Riviere de Canada ou de St Laurens et / tous les environs sont suivant les Relations des Francois. Par N. Sanson d'Abbeville Geographe ordinaire du Roy. / A Paris. / Chez Pierre Mariette Rue S Iacque a l'Esperance / Auecq priuilege du Roy, pour vingt Ans/.1656."

Mêmes remarques que pour la carte précédente, mais ici la partie qui nous intéresse est à plus grande échelle.

11. Creuxius, 1660. "Tabula Novæ Franciæ. Anno 1660".

Carte reproduite en hors-texte dans Crouse, Nellis M., Contributions of the Canadian Jesuits to the geographical knowledge of New France, 1632-1675. (Pour référence, voir No 1). Le lac Mistassini est à peu près sûrement celui nommé Outakouamicus lacus sur cette carte.

12. BlaEU, Johannes, 1662. Extrema Americæ / Versus boream, ubi / Terra Nova / Nova Francia, adjacentiaq. / Amsteledami Io: Blaeu Exc.

Carte d'un atlas de Blaeu datant de 1662. Le lac Mistassini n'est pas mentionné, mais au-delà du lac Saint-Jean se trouvent des lacs plus ou moins fantaisistes qui sont peut-être un rappel du lac Mistassini. Reproduit dans: "In the privy council. Maps and charts fyled as exhibits in the case Re Labrador Company, appellant, and the Queen, respondent, and E. Contra". (1891). Ce factum est généralement cité sous le nom de "Dossier de la cause de Mingan". Voici ce qu'écrit de ce cartographe le P. Delanglez:

"The founder of this firm, Willem Janszoon, signed Blaeu only once, he generally spelled his adopted name Blauw or Blauew, but his sons and grandsons wrote Blaeu". (Delanglez, El rio del Espiritu santo, New York, 1945, p. 86, note 16. Aussi Mid-America, vol. 26, p. 143, note 16, 1944.

13. Sanson, G., 1669. Amerique / septentrionale / par N. Sanson geographe Ord ${ }^{\mathrm{re}}$ du Roy / Reveue et changée en plusieurs endroits / suivant les mémoires les plus recents / par G. Sanson Geogr Ordinaire du Roy. / A Paris / chez Pierre Mariette, rue S. Iacques à l'Esperâce. / Avec privilege de sa mai.té pour 20 ans. / 1669. Reproduction photo-litho, ministère de la Justice, Ottawa, $59 \mathrm{~cm}$. x $40 \mathrm{~cm}$.

Pour région du lac Mistassini, voir commentaires du No 9. (Collection Knechtel).

14. Jolliet, Louis, 1674. Nouuelle Decouverte en plusieurs Nations dans la Nouuelle France en l'anne 1673 et 1674. Reproduite dans Trw aITES, Vol. 59, en face de p. 86, 1900. Dimensions de cette édition, $54.5 \times 39 \mathrm{~cm}$. Reproduit de la Revue de Géographie, février 1883. 
Grande carte en couleur de l'est de l'Amérique, simplement signee "Joliet". La péninsule québécoise est à peu près conforme à la réalité, quant au contour, mais le Saguenay est mal placé. Le lac Mistassini n'y est pas représenté; mais il y a lieu néanmoins de citer cette carte afin de faire le point, avant le voyage de Jolliet au lac Mistassini. Cette carte, dans Thwaites, accompagnait le recit intitule: "Le premier voyage qu'a fait le P. Marquette vers le nouveau Mexique \& comment s'en est formé le dessein." Le P. Marquette n'a pas 6erit cette relation. (Voir Delanglez, The "Recit des voyages et découvertes du Père Jacques Marquette" Mid-America, 26: 173-194; 211-258. 1944. Le P. Delanglez dementit que le texte est de Dablon).

15. Jolliet, Louis, 1679. "Cette carte montre le chemin que Louis Jolliet a fait / depuis Tadoussac iusqu'à la mer du Nord, dans la / Baye d'Hudson; et marque la vraye scituation / de la Baye et du district. ce qui est marqué de rouge / est le chemin par où il a esté / Fait a Québec en Canada/ le 8me nouembre 1679 / L. Jolliet". Reproduite dans: Pinart, A.-L. Recueil de cartes, plans et vues relatifs aux Etats-Unis et au Canada, New York, Boston, Montreal, Québec, Louisbourg, 1651-1731. Reproduits d'après les originaux manuscrits et inédits, etc. exposés à la bibliothèque nationale à l'oocasion du quatrième centenaire de la découverte de l'Amérique. Sous la direction de A.-L. Pinart. Paris, (Librairie américaine et coloniale, E. Dufossø) 1893.

Pour commentaires, voir Jolliet, 16. La carte décrite semble la carte $153 \mathrm{du}$ Vol. 388 de la Bibliothèque nationale, Paris. Cette carte appartenait a Michel-Antoine Baudrand (fiche P. Delanglez).

16. Jolliet, Louis, 1679. "Cette carte montre le chemin que Louis Jolliet (a fait) / de (puis) Tadoussac iusqu'à la mer du Nord, d(ans) la b(aye) d'Hudson, / et la vraye scituation de la baye et du d'estroit, / ce qui est marqué de rouge / est le chemin par ou il /a (et)é. : Fait à Quebec, / le $8 \mathrm{me}$ (no)embre (1)679. L. Jolliet." Copie ms. mal conservée, sur papier, 77 x $52 \mathrm{~cm}$., Archives canadiennes, Ottawa. Original conservé au Dépot des Cartes et Plans de la Marine (i.e. Archives du Service hydrographique), Paris, document 123-8-1.

Il existe au moins trois variantes de cette carte, toutes de la main de Louis Jolliet et de la même époque apparemment. Le P. Jean Delanglez devait les décrire toutes dans l'édition française de sa monographie de Louis Jolliet. L'une des copies, dont il m'a communiqué la description, porte la mention "marqué par des points" au lieu de "marqué de rouge" (Archives du service Hydrographique, 123-8- $\mathrm{l}^{\prime}$ ). (Voir aussi H ARRIsSE, 2).

17. Joliset, Louis, 1684. (Province de Québec et baie d'Hudson). "A Monseigneur. / A Monseigneur de la Barre Gouuerneur / et Lieutenant General pour le Roy en toute / l'étendue de la Nouuelle france. / Par son tres humble et tres obeissant, et tres fidelle seuiteur / et suiet / Jolliet / 1684." Carte ms. des archives du service hydrographique (Dépot des cartes de la marine), Paris. Dimensions de l'original, $65 \mathrm{~cm} . \mathrm{x} 42 \mathrm{~cm}$. 
Copie photographique aux Archives canadiennes, Ottawa. Le lac Mistassini porte le nom de lac Timagaming, surmonté d'un point d'interrogation.

18. JaIllot, Hubert, 1685. "Partie de la Nouvelle France / Dedie / A Monseigneur le Marquis de Seignelay, et Lonré, , Baron de Sceaux; conseiller du Roy, en tous ses Conseils / Commandeur, et Grand Tresorier de ses ordres. / Ministre. Secretaire d'Estat / et des Commandements de sa Majesté. / Par son tres-humble et tres Obeissant Seruiteur / Hubert Iaillot." "A Paris. Chez H. Iallot / proche les Grands Augustins, aux 2 Globes. Aveo Priuilege de S. Maj.té 1685." Dimension, $65 \mathrm{~cm}$. x $46 \mathrm{~cm}$.

Archives canadiennes, Ottawa. Le même auteur a publiê une carte presque semblable en 1696. Le lac Mistassini y porte le nom de Lac Timagaming. Cette carte est bas6e partiellement sur les cartes manuscrites de Louis Jolliet. Une autre carte, dédiée par le père Hennepin au roi d'Angleterre, Guillaume III, et couvrant la région de Tadoussac à la baie James, est өvidemment inspirée de la carte de Jolliet, probablement par l'intermédiaire de la carte de Jaillot.

19. Franquelin, Jean-Baptiste-Louis, 1688. "Carte / de l'Amerique Septentrionale / depuis le ...jusqu'au deg. de latt. \& environ / ...\&... deg. de longitude / contenant / les pays de Canada ou Nouvelle France / la Louisiane, la Floride, Virginie, Nlle / Suede, Nle Yorc, Nlle Angleterre, Aca-/die. Isle de Terre-neuve \&c. / . . . . . . . . . /. Par / Jean Baptiste Louis Franquelin Hydrographe du Roy / A Québec - en Canada."

La région du lac Mistassini, dit "lac Timagaming" est baséé sur les cartes de Jolliet. La carte de Franquelin est reproduite dans Crouse, 1. Il en existe aussi une copie fac-similé partielle $(36 \mathrm{~cm} \times 25.5 \mathrm{~cm})$ dans le factum du conseil privé relatif à la cause de Mingan. (Pour référence, voir no 12).

20. Anonyme, circa 1695. (Carte d'un missionnaire Jésuite). "Reduced facsimile of Ms missionnary map of country northwest of Lake St. John, P.Q., made circa 1695." Thw AITES, vol. 65, en face de p. 44. Original conservé au collège Sainto-Marie, Montréal.

Dans la partie superieure, cette carte se rend jusqu'au territoire des "mistassins", mais il n'y a rien de tracé pour cette région. Le lettrage original est remplacé, sur la carte reproduite par Thwaites, par des légendes éorites au clavigraphe.

21. Anonyme, ante 1700. Carte generale / du grand fleuve de / S. Laurens qui a esté / decouvert plus de 900 / lieues avant dans les / teres des Indes / occidentales /. No 328 de "Les raretez des Indes. Manuscrit du XVII siècle." Reproduit dans "Importants livres et manuscrits relatifs aux Ameriques... vente 18 et 19 juin 1934, Hotel des ventes de Paris." La carte est à la suite de la p. 92. Copie photostatique conservée dans les collections de la Socí́té historique du Saguenay, $43 \mathrm{~cm}$. x $34 \mathrm{~cm}$.

Le tracé du lac Mistassini ne ressemble à celui d'aucune autre carte. Carte n'ayant guère de valeur. (P. Delanglez). 
22. De L'Iste, Guillaume, 1700. "L'Amerique/ Septentrionale. / Dressé sur les Observations de Mrs de l'Academie Royale des Sciences. \& quelques autres, \& sur les Memoires les plus recens. / Par G. de l'Isle Geographe A Paris. / Chez l'Autheur sur le Quai de l'Horloge / Avec Privilege du Roy pour / 20. ans. 1700." Dimensions: $59 \mathrm{~cm} \times 45 \mathrm{~cm}$.

Archives canadiennes, Ottawa. Le lac Mistassini porte le nom de "L. des Mistasins". Contour inspiré des données de Jolliet, mais orientation du lac quelque peu différente, presque N.-S. "Toutes les cartes antérieures â 1704, signées par Guillaume Delisle, sont l'œuvre de son père, Claude". (Voir Delanglez, "The sources of Delisle map of América." 1703, Mid-America, 25 (1943): 275-298.

23. De r'Iste, Guillaume, 1703. "Carte / du Canada / ou de la / Nouvelle France / et des Découvertes qui y ont été faites / Dressée sur plusieurs Observations / et sur un grand nom de Relations imprimées ou manuscrites / Par Guillaume De l'Isle / de l'Academie Royale des Sciences / et Premier Geographe du Roy / A Paris / Chez l'Auteur sur le Quai de l'Horloge a 1 Aigle d Or / avec Privilege de sa Maj. pour 20. ans / 1703." Dimensions: $58 \mathrm{~cm} . \times 45 \mathrm{~cm}$.

Archives canadiennes, Ottawa. Une photo de la collection Karpinski (Archives publiques, Ottawa), intitulée Carte du Canada et du Misssissipi, par de l'Isle, 1702, est sensiblement la même que la carte de 1703. L'atlas italien, intitulé Atlante novissimo, de 1750, reproduit la carte de 1703 de De l'Isle, mais avec légende italienne. Sur la carte de 1703, le lac Mistassini porte le nom de "L. Timagaming ou L. Mistasin". Contrairement à la carte de 1700, l'orientation du lac Mistassini sur celle de 1703 est presque E.-W. La collection Knechtel renferme aussi les deux éditions suivantes de la carte de 1703: a) Portant la mention imprimée "A map illustrating the knowledge which French possessed of the Northern part of North America prior to the treaty of Utrecht'. Contour bleu. Le cartouche est un rouleau déroulé. $67 \mathrm{~cm}$. x $51 \mathrm{~cm}$. b) Portant la note imprimee: "Note the yellow and red lines indicate the claims of the English and French..." Photo-litho, Ministère de la Justice, Ottawa. Cartouche armoirie. $58.5 \mathrm{~cm}$. x $46 \mathrm{~cm}$.

24. Anonyme, circa 1703. Carte du Canada ou de la Nouvelle France, \& des découvertes qui y ont été faites. Dressée sur les observations les plus nouvelles \& sur divers mémoires tant manuscrits qu'imprimes. Dimensions de la copie photostatique, $52 \mathrm{~cm} . \times 41 \mathrm{~cm}$.

Carte faisant partie d'un mémoire imprimé ou d'un atlas, ainsi qu'en fait foi la mention "Tom VI No 20 Pag. 82" à la marge. Je n'ai pu toutefois l'identifier encore. La carte est apparemment basée sur celle de De l'Isle et le contour du lac Mistassini est celui de Jolliet. La carte porte un long texte sur le voyage de Robert Cavelier de la Salle. Lahontan également cité: "A moins que le dit Sr de Lahontan n'ait inventé toutes ces choses". La mention de Lahontan situe done l'édition de cette carte après 1703. (Collêtion Knechtel).

25. De Fer, N., 1705. "Le Canada, ou Nouvelle France, / la Floride, 
la Virginie, Pensilvanie, Caroline, / Nouvelle Angleterre et Nouvelle Yorck, / L'Isle de Terre Neuve, la Louisiane / et le Cours de la Riviere de / Misisipi.) Par N. de Fer, Geographe de Monseig. le Dauphin. / A Paris, / Chez l'Auteur dans l'Isle du Palais sur le Quay de l'Orloge à la Sphere Royale / Avec Privilege du Roy 1705." Dimensions: $34 \mathrm{~cm}$ x $23 \mathrm{~cm}$.

Archives canadiennes, Ottawa. Le lac Mistassini s'y nomme "Lac Timagaming". Seul le nom est inspiré des cartes de Jolliet; le tracê du lac, est absolument fantaisiste.

26. Thornton, Sam., 1709. [ Hudson's Bay and Labrador (Treaty of Utrecht)]. "Photo-lithograph from a photographic copy of an original map in the offices of the Hudson's Bay Company, London, England. The original is described in the Company's inventory of maps as follows: "No. 1 - Hudson's Bay and Labrador (Treaty of Utrecht)", The red line running southwestward from Grimington Island on the Atlantic Coast to lake Miscosink (Mistassini) and the accompanying legend, as well as the names, appear to identify this map as the one which accompanied the memorandum of the Hudson's Bay Company, addressed to the Lords Commissioners of Trade and Plantation, 7th February, 1711-12". Outre cette inscription moderne, imprimée, l'inscription ancienne, manuscrite: "Made by Saml Thornton at the Signe / of the Platt in the Minories / London Anno: / 1709". Dimensions de la copie photo-litho: $48 \mathrm{~cm}$. x $73 \mathrm{~cm}$.

Archives canadiennes, Ottawa. La cartographie de la région de Mistassini est plutôt fantaisiste.

27. Senex, John, 1710. North / America. / Corrected from the observations communicated to the Royal Society / at London and the Royal Academy / at Paris / by Iohn Senex / 1710. / To the Honorable Anthony Hammond/Esq. /One of the Commissionners of / Her Maj. ties Navy/This map is humbly dedicated and presented by his / Obliged Servant. (Collection Gagnon, Bibliothèque municipale, Montréal). Reproduit dans le dossier de la cause de Mingan. Pour référence, voir no 12). Dimensions de l'édition du dossier de Mingan: $46 \mathrm{~cm}$. x $34 \mathrm{~cm}$.

Le contour du lac "Mistasin" est basé sur la carte de De l'Isle, 1703.

28. Moll, Hermann, 1717. A map of America according to ye newest and most exact observation by Hermann Moll, geographer." In: "Atlas geographis, or a compleat system of geography (ancient and modern) for American" Vol. 5, 807 p. + 4, 1717.

Sur la carte citée, la péninsule québécoise se nomme "New Britain". Une rivière se rend du Saint-Laurent à la baie James, s'élargissant au centre en un lac non nommé et qui semble représenter le lac Mistassini. Inspirée apparemment de $N$. de Fer, 25.

29. Laure, Pierre, 1731. "Carte / du domaine en Canada / dediee / a / monseigneur le dauphin." A la suite d'une longue dédicace: "P. Laure J. / a chekoutimi le 23e d'aout / 1731". - ..."Cette carte des Mistassins depuis Piekouagami jusqu'a Nemiskau doit paroitre / d'autant plus fidelle que je l'ai faite avec l'aide des Srs Dorval - des Groisiliers, / petits Neveux 
du Sr Medatchouard, lesquels ont hyverne l'un 2 et l'autre 3 ans / avec succés aux grands Mistassins pour M.M. de la Compagnie d'occident." Dimensions de l'original: $82 \mathrm{~cm}$. x $56 \mathrm{~cm}$.

Original conservé à Paris, Bibliothèque du Service hydrographique de la Marine, ms, $4044-\mathrm{B}$, No 17. Reproduction, Archives canadiennes, Ottawa. La carte porte plusieurs inscriptions élaborées. Le contour du lac Mistassini, très compliqué (et fortement corrigé dans l'édition mentionnée à la suite), est à l'origine de la cartographie du lac depuis cette époque jusqu'à la publication de la carte de Low en 1885. Quelques cartes toutefois, n'ayant pas subi l'influence de Laure, sont des rejetons de la cartographie de Louis Jolliet.

30. Laure, P. Pierre, tracé de le moyne, 1731-1732. "Carte / du domaine du roy / en Canada / Dressée par le Pere Laure Missionnaire J. / 1731 Augmentée de nouveau revelle et corrigée avec grand soin. / en attendant un exemplaire complet l'automne 1732 / "Dimensions de l'original: $46 \mathrm{~cm}$. x $34 \mathrm{~cm}$.

Original conservé à Paris, Bibliothèque du Service hydrographique de la Marine, ms 4044-B, No 18. Reprodustion, Archives canadiennes, Ottawa. C'est un tracé entièrement remanié de la carte précédentê.

31. Laure, P. Pierre. Esquisse, 1731-1732. "Carte / du domaine du roy / en Canada / Dressé par le P. Laure Miss. Jes. / 1731 / augmenté de nouveau revelie et / Corrigee avec grand soin / en attendant un exemplaire complet / l'automne 1732."

Reproduction de l'original, Archives canadiennes, Ottawa. Cette carte peut être une première esquisse ayant servi au tracé de la carte précédente ou bien une copie simplifiée. Elle renferme peu de toponymes, en comparaison avec la précédente. D'autre part, comme il y a des variations importantes dans le tracé de la ligne de partage des eaux, cette esquisse représente peut-être simplement une étape de l'évolution des connaissances cartographiques du père Laure. Au sujet de cette carte, le chef de la section des cartes des Archives publiques m'écrit: "Original not easily identifiable; several of the Laure maps listed in catalogues of maps in French Archives have similar, but not exactly identical titles.

32. Laure, P. Pierre,1733. Carte du domaine du Roy en Canada dressée par le P. Laure miss. j. et dédiée en 1731 à Monseigneur le Dauphin. Augmentée de nouveau revue et corrigée avec grand soin par le même en attendant un exemplaire complet 1732. Guyot f. 1733. Carte en couleurs reproduite par La Rochemonteix, "Les Jésuites en Nouvelle-France". 64 cm. x 45.5.

Carte ressemblant beaucoup aux deux précédentes (Nos 30 et 31), mais mieux exécutéê.

33. Laure, P. Pierre, 1733. Carte / du / domaine du Roy / en Canada / Dedié / à / Monseigneur Le Dauphin / Par / le Pere laure Jesuite missionnaire / de ces endroits / mise au net et corrigée / sur de bons memoires / Par / le Sr Guyot President / du grenier a Sel de / Versailles 1733. Reproduit dans le 
dossier de la cause de Mingan (pour référence voir no 12) avec l'inscription suivante: "Photographie partielle d'une carte contenue dans un Atlas intitule "Amerique Septentrionale, cartes particulières". Cet Atlas se trouve à la bibliothèque du dépot des cartes et plans de la Marine a Paris." Dimensions de la copie: $64 \mathrm{~cm}$. x $34 \mathrm{~cm}$.

Le tracé de la région du lac Mistassini se compare à celui des cartes 30, 31 et 32. (Collection Gagnon).

34. Anonyme, circa 1733. (Partie du domaine du roi). Grande carte sans titre ni date, mais semblant du père Laure. Photolitho of a map in the library of Parliament, Ottawa. Carte sans encadrement. Dimensions de la copie (ne tenant compte que du trace): $99 \mathrm{~cm} . \times 57 \mathrm{~cm}$.

Collection Knechtel.

35. Lahontan, Baron de, 1735. A general map of New France Com. call'd Canada. Vol. 1, p. 1 A. D'après une note ms de Voorhis, cette carte vient de l'Gdition anglaise de Lahontan $(1735.35 \mathrm{~cm} . \times 22 \mathrm{~cm}$. Dimensions de la copie photographique).

Collection Knechtel. Le lac Mistassini n'est pas représenté, mais par contre le fort de Mistassini est mentionné.

36. Anonyme, 1741. Carte Abrégée du / Canada / levée sur les lieux / par M. XXX / résident à Quebec / année 1741.

Carte reproduite dans le dossier de la cause de Mingan (pour rëférence voir no 12). Dimensions de la copie: $18 \mathrm{~cm}$. x $13.5 \mathrm{~cm}$. Le contour du lac Mistassini est vaguement basé sur les cartes de Laure de $1731-32(30,31)$.

37. Bellin, Jacques Nicolas, 1744. "Carte / de la partie Orientale / de la Nouvelle France / ou du Canada / dédiée / A Monseigneur le Comte de Maurepas / Ministre et Secretaire d'Etat, / Commandeur des Ordres du Roy. / Par N. Bellin Ingénieur de la Marine 1744."

Copie photo-litho, Archives canadiennes, Ottawa. Dimensions de la copie: $55 \mathrm{~cm}$. $\times 40 \mathrm{~cm}$. Le lac se nomme lac des Mistassins. Carte inspirée de la première carte de Laure. Une autre carte de Bellin, datant de 1755, $(55 \mathrm{~cm} . \times 43 \mathrm{~cm}$.), de même qu'une autre du même auteur, parue dans l'Atlas de Clouet, la même année $(43 \mathrm{~cm}$. x $55 \mathrm{~cm}$.), sont sensiblement des répétitions de la carte de 1744 de Bellin.

38. Hasius et Bofhmius, 1746. Americæ mappa generalis... secundum mentem Dim. Hasii M.P.P. ... et delineata ab Aug. Gottl. Bœhmio... 1746. Photo-litho, library of Congress, Washington. $55 \mathrm{~cm} . \times 47 \mathrm{~cm}$.

Sur cette carte, le lac Mistassini n'est plus qu'une baie de la baie James. Collection Knechtel.

39. D'Anvilue, 1755. Canada Louisiane et terres angloises par le $\mathrm{Sr}$ d'Anville de l'Academie Rle des inscriptions et Belles-Lettres et de celle des sciences de Petersbourg. Secrétaire de S.A.S. Mgr le duc d'Orleans, Novembre 1755. Sous le privilege de l'Academie. Feuillet nord de la carte: $115 \mathrm{~cm}$. x $56 \mathrm{~cm}$.

Le contour du lac Mistassini est basé sur la carte de Laure de 1731-32 (Laure, 30). Collection Knechtel. 
40. Huske et Kitchin. A new and accurate map of North Ameriea (wherein the errors of all preceeding British, French and Dutch maps, respecting rights of Great Britain, France and Spain \& the limits of each of his majesty's provinces are corrected). Humbly inscribed to the honorable Charles Townshen, one of the Right honorable Lords commissioners for executing the office of lord High Admiral of Great Britain \&c. By his most obliged, most obedient and very humble servant. Huske. Tho: Kitchin Sculpt Published for the present state of North America 7c and sold by R. \& I. Dodsley in Pall-Mall 1755. Photo-litho, Ottawa, $51 \mathrm{~cm} . \times 41 \mathrm{~cm}$.

Le contour du lac Mistassini est basé sur les cartes du père Laure. Collection Knechtel.

41. Anonyme, circa 1761. Plate I. "The claims of the French in 1756 are coloured yellow." (Copie de la section centre ouest d'une carte antérieure). Plate II. "The French Dominion as proposed by M. de Bussy in 1761 are coloured yellow and the lands proposed by $M$. de Bussy to be Neutral are coloured Green." (Copie centre ouest d'une autre carte antérieure). Photo-litho, Washington. Chaque planche mesure $32 \mathrm{~cm}$. x $27 \mathrm{~cm}$.

Collection Knechtel. Cartes sans titres préparées pour le traite de paix de 1763 ou celui de 1783, consacrant l'indépendance américaine.

42. Rocque, John, circa 1761. A general map of North America in which is express'd the several new roads, forts, engagements, Sc taken from actual surveys and observations made in the army emply'd there from the year 1754 to 1761. Drawn by the late John Rocque, topographer to his majesty. Cartes generales de l'Amerique septentrionale, en lesquelles sont exprimés les nouvelles Routes, Forts, Batailles Sc. d'apres les Observations qui ont été faites depuis l'an 1754, jusqu'à l'an 1761. Recueillies par feu Jean Rocque, topographe de sa majesté britannique, Sc. (Section N.E. de la carte). Photo-lithograph from copy of a map in the Department of Justice, Ottawa, $66 \mathrm{~cm}$. x $13 \mathrm{~cm}$. Laure.

Collection Knechtel. Contour du lac Mistassini d'après les cartes de

43. Bellin, Jacques Nicolas, 1763. "Baye de Hudson et Pays Voisins". Carte No 3, Tome I, de : "Le petit atlas maritime. Recueil de cartes et plans des quatre parties du monde en cinq volumes. Par le S. de Bellin, ingénieur de la marine, 1764." Dimensions de l'original: $34 \mathrm{~cm}$. x $22 \mathrm{~cm}$.

Archives canadiennes, Ottawa. La carte elle-même était datée de 1763 et le volume de 1764. Les deux cartes suivantes, du même atlas, ne portaient elles-mêmes aucune date. Le contour du lac Mistassini sur la carte de 1763 est inspiré de la première carte du père Laure. (Voir Laure, 29). Le lac se nomme "lac des Mistassins".

44. Bowen and Gibson, circa 1763. An accurate map of North America describing and distinguishing the British, Spanish and French dominions in this great continent. Exhibiting the present seat of war, and the French encroachments. Also all West Indian Islands belonging to and possessed by the several European princes and states. The whole laid down according 
to the latest and most authentick improvements by Eman Bowen. Geogr. to his majesty and John Gibson Engraver. L'édition consultée de cette carte porte en outre la mention: "This map is a reproduction of the map annexed to the report of 8th June 1763, from the Lords of Trade and Plantations to the King." $82 \mathrm{~cm} . \times 51 \mathrm{~cm}$.

Collection Knechtel. Le feuillet cité est une copie du secteur Nord-est de la carte originale. Contour du lac Mistassini d'après la carte de Laure, $1731-32$.

45. Bowen and Gibson, circa 1763. An accurate map... in this great continent. According to the definite treaty concluded at Paris $10^{\text {th }}$ Feb. ${ }^{\mathrm{y}}$ 1763. Also all the West India... (La partie omise du titre repète celui de la carte 44). $118 \mathrm{~cm} . \times 71 \mathrm{~cm}$.

La présente carte, basée sur lẹs mêmes tracés que la précédente, est le secteur nord d'une carte plus étendue.

46. Beluin, Jacques Nicolas, 1764. "La Nouvelle France ou Canada". Carte No 4, Tome I, de : "Le petit atlas maritime... 1764". (Voir précédemment pour référence). Dimensions de l'original: $35 \mathrm{~cm} . \mathrm{x} 20 \mathrm{~cm}$.

Archives canadiennes, Ottawa. Le contour du lac Mistassini est bas6 sur la première carte du père Laure (Voir LaURe, 29). Le lac se nomme "lac des Mistassins".

47. Bellin, Jacques Nicolas, 1764. "Cours du fleuve de Saint Laurent depuis la Mer jusqu'à Québec". Carte No 5, Tome I, de: "Le petit atlas maritime... 1764." (Voir BeLlin, 43, pour référence complète). Dimensions de l'original: $35 \mathrm{~cm}$. $21 \mathrm{~cm}$.

Archives canadiennes, Ottawa. Bien que cette carte soit à la suite des cartes nos 43-46, dans l'Atlas, son contour du lac Mistassini est basé non pas sur la première carte du père Laure (comme les cartes 43 et 46), mais sur celles de 1731-1732 (Ladre, 30, 31). Le lac se nomme "Lac des Grds Mistassins".

48. KItchin, Thomas, 1764. "A New Map of the Province of Quebec in North America; Drawn from the best Authorities: By Tho" Kitchin Geogr." Dimensions de l'original: $22 \mathrm{~cm} . \times 17 \mathrm{~cm}$.

Archives canadiennes, Ottawa, et Collection Gagnon, Bibliothèque municipale, Montréal. La date n'est pas inscrite toutefois sur cette carte, dessinee "For the London Magazine", comme l'indique une mention sur la carte. Le contour du lac Mistassini est celui des cartes de Laure de 17311732 et semble s'inspirer à la fois de ces cartes et de la carte No 5 de Bellin, 1764. (Voir Bellin, 47). Le lac se nomme sur la carte de Kitchin "Mistassin L".

49. Bell, Peter, 1772. A map of the British dominion in North America according to the treaty in 1763 by Peter Bell, geographer, 1772. Photolithograph copy of a map in the Library of Congress, Washington. $40 \mathrm{~cm}$. x $30 \mathrm{~cm}$.

Collection Knechtel. Le contour du lac Mistassini est basé sur les cartes de Laure de 1731-32 (LAURe, 30, 31).

50. Carver, circa 1776. "A new map of the province of Quebec 
according to the Royal proclamation, of the 7th of October 1763, from the French Surveys connected with those made after the war, by Captain Carver and other officers, in his Majesty's service." Dimensions de l'original: $67 \mathrm{~cm}$. x $49 \mathrm{~cm}$.

Collection Gagnon, Bibliothèque municipale, Montréal, et Societé historique du Saguenay. La carte ne porte aucune date, sauf celle inscrite par le collectionneur. Cette carte ne couvre pas le territoire du lac Mistassini, mais la région immédiatement au sud, entre ce lac et le lac St-Jean. Cette partie de la carte est inspirée de celles de Laure.

51. Jefferys, Thomas, 1776. Extract from the American Atlas by the late M. Thomas Jefferys, Geographer to the king etc. 1776. Reproduit dans le dossier de la cause de Mingan. (pour référence, voir 12). 19cm. large x $26 \mathrm{~cm}$. haut.

Contour du lac Mistassini basé sur les cartes de Laure 1731-32. (LA URE, $30,31)$.

52. Carver, 1778. A new map of North America from the latest discoveries, 1778. Engrav'd by for Carvers Travels. Photo-lithograph copy of a map in the library of Congress, Washington. $36 \mathrm{~cm} . \times 33.5 \mathrm{~cm}$.

Collection Knechtel. Région du lac Mistassini basée sur la première carte de Laure (LAURe, 29).

53. Universal Magazine, prob. 1781. "A new and accurate Map of Quebec and its Boundaries from a late Survey." The Universal Magazine (Londres). Sept. 1781. Dimensions de l'original: $31 \mathrm{~cm} . x 27 \mathrm{~cm}$.

Carte détachée, sans date, conservée dans la collection Gagnon, Bibliothèque municipale de Montréal. Il n'y a sur la carte aucune référence originale permettant de l'identifier, sauf l'inscription du collectionneur. Attach 6 à cette carte la coupure suivante du Universal Magazine: Anonyme, "A geographical description and natural history of the province of Quebec, in North-America; with an accurate whole sheet map of the country." The Universal Magazine for September, 1781, pp. 113-116. Dans une note infrapaginale de cet article, on peut lire: "In our Magazine for February, 1761, is an accurate map of Canada, with its boundaries, before the Royal proclamation in 1763, and the Quebec Bill in 1774 took place." La carte de la Collection Gagnon pourrait être à mon avis plutôt celle de 1781, mentionnée dans le titre de l'article cité, que celle de 1761, mentionnée en note infrapaginale; sans compter que sur la carte de l'Universal Magazine se trouve une ligne frontière de la province de Québec, vaguèment parallèle au St-Laurent et traversant le lac St-Jean, telle qu'on la trouve sur une carte de Carver (CARver, 50) citée plus loin, et fixée par la proclamation royale du 7 octobre 1763. Le contour du lac Mistassini est basé sur les cartes de Laure de 17311732 (LA URE, 30, 31) et le lac se nomme "lake Mistassini".

54. SAYER, Robert, 1788. "A new and correct map of the British colonies in North America comprehending Eastern Canada with the province of Quebec, New Brunswick, Nova Scotia, and the Government of Newfoundland: with the adjacent states of New England, Vermont, New York, 
Pennsylvania and New Jersey. London. Publish'd by Robert Sayer, 53 Fleet street, $1^{\text {st }}$. Jan. ${ }^{y}$ 1788." Dimensions de l'original: $67 \mathrm{~cm} . \times 48 \mathrm{~cm}$. Archives canadiennes, Ottawa. La cartographie de la région entre le lac St-Jean et le lac Mistassini est basée sur les cartes de Laure de 17311732." Le lac se nomme "Mistassins lake" (LAURE, 30 et 31).

55. Laurie \& Whittles, 1788. A new and correct map of the province of Quebec with adjacent states and provinces from the French surveys, connected with thoses made by Captain Carver and other officers. 1 J.an 1788. Dimensions $68 \mathrm{~cm}$. $x 50 \mathrm{~cm}$.

Archives canadiennes, Ottawa. Cartographie de la région du lac Mistassini basée sur celles de Laure. Cette carte est pratiquement une réédition de Carver, 50.

56. Mackenzie, Alexander, 1798. "Carte de la partie de l'Amérique comprise entre le $40^{\mathrm{eme}}$ et le $70^{\mathrm{eme}}$ Degrés de Latitude Nord et le $45^{\mathrm{eme}}$ et $180^{\text {eme }}$ Degrés de Longitude Occid. le où l'on a tracé la Route d'Alexandre Mackenzie, Depuis Montréal au Fort Chipiouyan et à la Mer Glaciale en 1789 et du fort Chipiouyan au Grand Ocean Boreal en 1798." In: Mackenzie Alexandre, Voyages d'Alexd ${ }^{\text {re }}$ Mackenzie dans l'intérieur de l'Amérique septentrionale, faits en 1789,1792 et 1793 . 3 vol., 409,420 et 388 pp., 3, carte hors texte (dans vol. 3), Paris (Dentu, Imprimeur libraire), An X-1802.

La cartographie du "lac Mistissinny" est inspirée de Laure.

57. Boucheate, Joseph, 1815. Map of the Provinces of Upper \& Lower Canada with the adjacent parts of the United states of America. Compiled from the latest surveys and adjusted from the most recent and approved astronomical observations by Joseph Bouchette. 1815". Dimensions de l'original: $1 \mathrm{~m} .22 \times 0 . \mathrm{m} .76$.

Archives canadiennes, Ottawa. Le contour du lac "Mistissinny" tire son origine de la cartographie de Laure.

58. Political Magazine, 1832. "Lower Canada and New Brunswick". Carte publiée en 1832 par le Political Magazine (published by Baldwin and Cradock).

Archives canadiennes, Ottawa. Le contour du lac Mistassini est base sur la cartographie de Laure.

59. Arrowsmith, J. 1832. British North America by permission dedicated to the Hon. ble Hudson Bay Company containing the latest information which their documents furnish. By their obedient Servant J. Arrowsmith. London. Publ. 15 feb. 1832. Photo-lithograph copy of a map in the Library of Congress, Washington. $59.5 \mathrm{~cm} . \times 48 \mathrm{~cm}$. 1834".

Collection Knechtel. Voorhis a écrit en marge de la carte la date "April

60. Oliver \& Boyd, 1839. To accompany "British America". Vol. 1, 1839. Published by Oliver \& Boyd, Edinburgh. $56 \mathrm{~cm} . \mathrm{x} 26.5 \mathrm{~cm}$.

Collection Knechtel. Le lac Mistassini, très allonge et renfermant une grande île au centre, n'est inspiré ni de Jolliet, ni de Laure.

61. Arrowsmith, J., 1857. Map of North America. Drawn by J. 
Arrowsmith. On this map the territories claimed by the Hudson's bay company in virtue of the charter granted to them by King Charles the second are coloured Green. The other British territories Pink \& those of Russia yellow. London, $1857.53 \mathrm{~cm}$. x $40.5 \mathrm{~cm}$.

Collection Knechtel. Le poste de Mistassini est indiqué sur la carte.

62. Hind, Henry Youle, 1863. Map of the Peninsula of Labrador showing the canoe route from Seven Islands to Hamilton Inlet." In: HIND, Henry Youle, Explorations in the interior of the Labrador Peninsula. Londres, 1863.

63. Bronet, abbe Ovide, 1864. Michaux and his journey in Canada. $\mathrm{Ca}$ nadian Naturalist, New Series, 1: 325-337. 1864. Aussi tirage à part $13 \mathrm{p}$. 1864. Renferme une carte intitulee: "Map of the country between lake St. John \& Lake Mistassini. Compiled by the abbe O. Brunet. 1863." $30 \mathrm{~cm}$. x $19 \mathrm{~cm}$.

Carte tracée d'après celles du père Laure (Nos 30-33). Brochure traduite et légèrement abrégఠe, avec permission de l'auteur par T.S.H. [ T. Sterry-Hunt ]. L'édition française, parue en 1861, ne renfermait pas de carte.

64. TACHÉ, Eugène, 1870. Carte de la province de Québec, Canada, dressée au Département des Terres de la Couronne. Par Eugène Taché, Assistant Commissaire, Québec, 1870." Dimensions de l'original, 1 m. 35 x 0 m. 94 .

Collection Gagnon, Bibliothèque municipale, Montréal. Cette carte ne couvre pas la région du lac Mistassini, mais elle porte, dans la partie supérieure de la rivière Mistassini, cette inscription: "Cette Rivière est supposé être l'ancienne route conduisant au Grand Lac Mistassini ou des Misstasins."

65. Genest, P.M.A., 1875. "Carte de la Nouvelle France Pour servir à l'étude de l'Histoire du Canada. Depuis sa Découverte Jusqu'en 1760. Par P.M.A. Genest. Enregistré conformément à l'Acte du Parlement du Canada, en mil huit cent soixante-et quinze, par P.M.A. Genest, au bureau du Ministre de l'Agriculture. Imprimé par la Compagnie de Lithographio Burland-Desbarats, 319 Rue St. Antoine, Montréal." Dimensions de l'original: $1 \mathrm{~m} .50 \times 0 \mathrm{~m} .92 .40 \mathrm{mi}$. au pouce.

Archives canadiennes, Ottawa. Le contour du "lac des Mistassins" tire peut-être, partiellement, son origine de la cartographie de Laure. Il peut être aussi fantaisiste ou d'une source qui m'est inconnue. Dans Unrivalled Atlas (1878), se trouve une carte qui semble basée sur celle de Genest. (Archives publiques, Ottawa).

66. TACHÉ, Eugène, 1880. "Carte de la province de Québec, Canada, dressée au Département des terres de la Couronne par Eugène Taché, Assistant Commissaire, Québec. 1880." Dimensions de l'original: 1 m. 35 x 0 m. 94.

Collection Gagnon, Bibliothèque municipale, Montréal. La cartographie de la région du lac Mistassini, basée sur les relevés préliminaires de la Commission géologique d'Ottawa, commence à se préciser. 
67. Beld, Robert, 1880. [ Région de la baie James et de la baie d'Hudson ]. Carte illustrant l'étude suivante: Bell, Prof. R., Recent explorations around Hudson's bay. Bull. Soc. Géog. Québec, 1 (No 1): 30-42. 1880.

Dans le texte, rien sur la région du lac Mistassini, mais la carte couvre ce territoire. Le contour de la partie sud-ouest du lac Mistassini, comme la carte de Tache de 1880 (voir 66), tient compte des explorations de la Commission géologique d'Ottawa, exécutées par Richardson et MoOuat.

68. ANONYME ET SANS DATE. "Plan of part of the North shore of the St. Lawrence from James Bay to the Mingan Mainland."

Archives canadiennes, Ottawa (T. 53). La région du lac Mistassini est basée sur la cartographie de Laure.

\section{$* *$ \\ C. Anciennes Cartes manuscrites}

\section{A RECHERCHER}

69. Clouston, James, 1821-24. A.P. Low, dans "Report on explorations in the Labrador peninsula along the East Main, Koksoak, Hamilton, Manicuagan and portions of others rivers in 1892-93-94-95" (Geological Survey of Canada, Annual Report, Vol. VIII, Part L, Ottawa 1896), écrit: "Between 1821 and 1824, James Clouston was employed in exploring the country east of Hudson Bay. There are no available notes or records of his travels, and all that remains is a map on a small scale, showing the routes that he followed. These embrace the East Main river to the Tichagami branch, a few miles beyond the old post of Birch Point, two portage-routes between the East Main and Rupert rivers, the Rupert river and lake Mistassini, and the routes to Waswanipi on the Nottaway river. The original map is at Great Whale River post, where a tracing of it was made in 1888, which is now in the Geological Survey office."

J'ai vainement cherché à Ottawa, tant dans les collections de la Commission géologique qu'aux Archives nationales, la copie de la carte de Clouston dont parle Low. Quant à l'original, il n'est sûrement plus au poste de Great Whale River, les archives des postes étant périodiquement transférées aux Archives centrales de la Hudson's Bay Co., à Londres. Je ne pourrai avoir le renseignement à temps pour en faire part ici.

70. Beads, John, and Spenser, John, 1842. Dans l'ouvrage de Low précité (voir no 69), l'auteur écrit: "In 1842, John Beads and John Spenser, at Nichicun and lake Kaniapiskau, compiled a map of the region surrounding these places, giving the various branches and lakes of the rivers draining southward, westward and northward, from the central portion of the peninsula. This map was found at Nichicun in 1893, and is now in the Geological Survey office. It has been used largely in the compilation of the unsurveyed parts of the map accompanying this report."

Je n'ai pu trouver cette carte manuscrite, ni dans les collections de la Commission géologique, ni aux Archives nationales, à Ottawa. 
71. Galbraith, John. 1885. "Canœ-trip from lake Superior to Hudson bay and return via lake Mistassini and the Saguenay river to Tadoussac." Bull. Soc. de Géog. Québec, 1, no 4: 1885. 1-12. Une carte devait accompagner son texte, mais la rédaction du Bulletin a dû en renvoyer la publication à un numéro ultérieur, ce qui n'a jamais été fait.

Je n'ai pu savoir si la carte manuscrite se trouvait encore dans les archives de la Société de Géographie de Québec.

\section{Cartographie moderne}

Les cartes sont citées dans l'ordre chronologique et celles sans date ont été datées approximativement. Ce sont les Numéros 89, 90, 91, 92. Dans cette section, il a fallu se limiter aux cartes apportant des données nouvelles, vraies ou fausses. Je n'ai pu citer toutes les éditions des cartes officielles, ni toutes les cartes figurant dans des ouvrages relatifs à la région. Ces travaux sont d'ailleurs cités dans les autres sections de ma bibliographie manuscrite de la région. J'aj da omettre également les manuels scolaires qui, forcément, se contentent de reproduire des décalques, souvent déformés, de la cartographie officielle. Je reconnais toutefois que plusieurs autres cartes et atlas pourraient enrichir cette liste.

72. Low,A.P., 1885. "Map of lake Mistassini with geological boundaries to illustrate the report of A.P. Low, B. Ap. Ac. Scale: 8 miles to 1 inch. 1886." Dimensions: $37 \mathrm{~cm}$. $x 37 \mathrm{~cm}$.

Faisant partie de: Low, A.P., Report of the Mistassini expedition, 1884-85. 55 p. Part D, Annual Report, 1885, Geological and Natural History Survey of Canada. Ce rapport a également été traduit en français, mais je n'ai pas vu cette édition. Bien que le rapport soit daté de 1885 , la carte est indiquée comme étant de 1886. Première carte donnant, avec assez de précision, le contour du lac Mistassini. Le dessin des îles du centre est néanmoins fantaisiste et le contour du lac Albanel, approximatif.

73. Bignell, John, 1885. [ Plan d'une partie de la rivière Témiscamie ]. "Plan R. 34-B-1, par John Bignell, 1885. $1 \mathrm{mi}$. au pouce." Plan ms., Service des Arpentages, Ministère des Terres et Forêts, Québec.

74. Eaton, D.I.V. "Map of Labrador Peninsula, South West sheet. Natural scale 1/1,584,000. Scale: 25 miles to one inch. Compiled by D.I.V. Eaton, C.E. Drawn for photo-lithography by C.O. Senécal, C.E. - Accompanying Report by A.P. Low, B. Ap. Sc., Part L, Vol. VIII, (New Series) Geological survey of Canada, 1896."

Cette carte, qui comprend quatre feuillets (et dont je n'ai cité que le feuillet sud-ouest), accompagne le "Report on exploration in the Labrador 
Peninsula along the East Main..." (Pour référence, voir No 69 supra). Bien que le portefeuille renfermant les cartes soit daté de 1895 , les cartes elles-mêmes sont datées de 1896.

75. O'Sullivan, Henry, 1895. "Map of an exploration of part of the Hudson Bay slope and of a survey of the adjacent waters of the Upper Ottawa region performed by Henry O'Sullivan, D.L.S., under instructions from the Department of Crown Lands. Dated Quebec, 6 october 1891; 6 december 1892; 15 september 1893; 16 august 1894. Being in continuation of those issued on the 30 sept. 1890. - Quebec, 22nd October 1895." Echelle, 12 milles au pouce. $20 \mathrm{~cm} . \times 36 \mathrm{~cm}$. Carte hors texte accompagnant: Rapport préliminaire sur l'exploration de l'étendue de pays comprise entre le lac St-Jean et la baie de James faites (sic) en vertu d'instructions du département de la colonisation et des mines de la province de Québec. Par Henry O'Sullivan, D.L.S. \& C.E. Ingénieur civil et inspecteur des arpentages, P.Q." Documents de la session No 111, 61 Victoria, A.D. 1898. Québec 1898.

Cette carte ne se rend qu'au lac Waswanipi et ne couvre pas la région immédiate du lac Mistassini. La carte est datée de 1898, la lettre liminaire d'O'Sullivan de 1897, et la publication de 1898.

76. O'Sullivan, Henry, 1900. "Carte d'une route conduisant du lac St-Jean à la baie James par les rivières Chamouchouan, Nottaway et Rupert exploree en 1897-98-99 par Henry O'Sullivan A.D., A.P., et I.C., inspecteur des arpentages de la province de Québec. - Map of route from lake St. John to James bay via Chamouchouan, Nottaway and Rupert rivers surveyed in 1897-98-99 by Henry O'Sullivan, D.L.S., P.L.S., and C.E., inspector of surveys for the province of Quebec." Département des Mines et de la Colonisation, Québec, 30 juin, 1900. Echelle, 12 milles au pouce. $74 \mathrm{~cm}$. $\mathrm{x}$ $63.5 \mathrm{~cm}$. Carte hors texte accompagnant le "Second report of progress of exploration in the country between lake St. John and James Bay. By Henry O'Sullivan... department of Colonization and Mines, Québec 1901.

77. Laut, Agnes C. "Northwest territories". Carte faisant partie de l'ouvrage de Laut, The conquest of the Great Northwest, New York (Moffat, Yard and Co.), 1914.

78. Denis, Thé-C., 1915. "Map of New Quebec territory (formerly district of Ungava) province of Quebec, geologically coloured from explorations by A.P. Low and Robert Bell, Reports and maps of Geological Survey, Ottawa. Province of Quebec, Canada, Department of Colonization, Mines and Fisheries, Mines branch... Théo. C. Denis, Superintendent of Mines, 1915. Scale, 1: 2,217,600 or 35 miles to 1 inch. Base map from plates of Map of Dominion of Canada, Dept of Interior, Ottawa. J.E. Chalifour, Chief Geographer." Carte hors texte, $79.5 \times 63 \mathrm{~cm}$., accompagnant l'ouvrage compilé par Théo. C. Denis, intitulé: "Extraits de rapports sur le district d'Ungava récemment annexé à la Province de Québec et constituant le Nouveau Québec. Ministère de la Colonisation, des Mines et des Pêcheries, Québec,1915." 
Une première édition de l'ouvrage renfermant cette carte a paru en 1913, mais moins considérable. J'ignore s'il y avait une carte. Une autre Gdition a paru en 1929. La carte accompagnant cette dernière $(83 \mathrm{~cm} . x 88 \mathrm{~cm}$.) avait été dessinée de nouveau et mise à jour. Echelle également 35 milles au pouce. Le titre est le suivant: "Carte geologique du Nouveau Québec (autrefois district d'Ungava). Des rapports et cartes de la Commissiongéologique, Ottawa. - Geological map of the New Quebec Territory (formerly district of Ungava). From reports and maps of the Geological Survey, Ottawa". Cette dernière carte a été faite sous la direction de A.-O. Dufresne.

79. Speck, Frank G., 1923. "Map of the region of Lake Mistassini showing the hunting territories of the Mistassini Indians." 50 milles au pouce. $21 \mathrm{~cm}$. $\times 19.5 \mathrm{~cm}$. Hors texte accompagnant l'étude de Speck, "Mistassini hunting territories in the Labrador peninsula", American Anthropologist, Oct.-Dec. 1923.

80. Crouse, Nellis M., 1924. "Routes to Hudson bay". Hors texte (33 cm. x $30 \mathrm{~cm}$.$) accompagnant l'ouvrage de Crouse précédemment cité$ (voir No 1).

L'auteur donne un tracé des routes conduisant de la vallée du SaintLaurent à la baie d'Hudson. Des quatre routes passant par le lac Mistassini, il ne mentionne pas la rivière Mistassini. Pour description de cette dernière route, voir No 113, Rousseau. Le trace de Crouse est fait sur une carte publié par Geographia Ltd, 55 Fleet Street, London.

81. Marie-Victorin, F., 1925. "Tracé général de l'itinéraire de Michaux, de Tadoussac à la rivière Rupert". Dans Marie-Victorin, Études floristiques sur la région du lac Saint-Jean Fig. dans le texte.

81a. Marie-Victorin, F., 1925. "Reproduction d'une partie de la "Carte du domaine du Roy en Canada, dressée par le P. Laure, miss. j., et dédiée en 1731 à Monseigneur le Dauphin," permettant l'interprétation de quelques points obscurs de l'itinéraire de Michaux." Fig. dans le texte. Dans l'étude précitée du F. Marie-Victorin.

Cette carte fait l'objet de discussion dans Rousseau, No 113.

82. WHiтE, James, 1926. "Forts and trading posts in Labrador peninsula and adjoining portions of Ontario and Quebec. Compiled by James White, F.R.G.S." Dimensions: $58 \mathrm{~cm}$. x $47 \mathrm{~cm}$. 60 milles au pouce. Hors texte de l'ouvrage de White, cité plus haut, No 6.

83. Willson, B., copie Voorhis, 1926. "Map of the company's territory. Enlargment of map in the "History of the Great Company" by B. Willson (1899)." Carte portant la note ms. de Voorhis: "This map probably represents the company's forts about 1840." $49 \mathrm{~cm}$. x $38 \mathrm{~cm}$.

Collection Knechtel.

84. Burpee, Lawrence J., 1927. An historical atlas of Canada. 48 p., 83 cartes, (Thomas Nelson) Toronto, 1927.

Plusieurs cartes de cet atlas intéressent la région du lac Mistassini. Ce sont celles qui donnent les anciennes frontières de la Nouvelle-France, les cartes des productions naturelles, etc. 
85. Voorhis, E., 1927. "French forts and fortified posts 1608-1763, E.V." Natural resources intelligence Service, Ottawa, $1927.45 \mathrm{~cm}$. x $52 \mathrm{~cm}$. haut.

Collection Knechtel.

86. Canadian National Railways, 1928. "Canœ trips \& Nature photography", 1928. Renferme, dans le texte, les cartes suivantes: "Lake St John to lake Mistassini trip", "Lake Mistassini to Rupert House, James bay canœ trip".

Chaque carte accompagnée de courts commentaires sur le trajet.

87. BÉlanger, Henri, 1929. "Plans faits sous la direction de Henri Bélanger, arpenteur, et remis au Ministère des Terres et Forêts en 1929." a) Plan du lac Mistassini; b) plan du lac Albanel; c) lac Chibougamau aux lacs Doré et Waconichi. 40 chaînes au pouce.

Plans manuscrits conservés au Service d'Arpentage, Ministère des Terres et Forêts, Québec. La dernière carte citée va du lac Chibougamau à la baie du Poste du lac Mistassini. Ces plans sont les plus importants documents cartographiques sur la région.

88. Ministêre des Terres et Forêts, Qú́bec, 1929. "Route de Chibougamau de la gare d'Escalana sur le chemin de fer Canadien National au lac Chibougamau." $3 \mathrm{mi}$. au pouce. Service des Arpentages, Département des Terres et Forêts, Québec, 1929.

Carte-guide, en deux feuillets, sous couverture, pour les excur sionnistes. Le territoire couvert se rend au lac Waconichi.

88a. Dufresne, A.-O., 1929. Voir No 78, Denis.

89. White, James, circa 1930. "Map of the dominion of Canada showing the establishments of the Hudson's Bay Company. Natural scale 1/6,336,000. Scale 100 miles to one inch. James White, F.R.G.S., geographer. $91 \mathrm{~cm} . \times 51 \mathrm{~cm}$.

Collection Knechtel.

90. Voorhis, E., circa 1930."Chief forts and trading posts of the Northwest Company and explorers, $1820 . " 89 \mathrm{~cm}$. x $49 \mathrm{~cm}$.

Collection Knechtel. Carte No 2 de Voorhis. Le poste de Mistassini y est situé à l'endroit où il se trouve présentement. Carte basée sur celle du Natural Resources Intelligence Service.

91. Voorhis, E., circa 1930. "Hudson's Bay Company forts and trading posts, 1670 to 1820 ." $89 \mathrm{~cm}$. x $49 \mathrm{~cm}$.

Collection Knechtel. Le poste de Mistassini est situé près de la rivière Rupert. Carte No 3 de Voorhis, basée sur celle du Natural Resources Intelligence Service.

92. Voorhis, E., circa 1930. “Hudson's Bay Co's Forts. 1872." $89 \mathrm{~cm}$. x $49 \mathrm{~cm}$.

Collection Knechtel. Carte No 5 de Voorhis. Basee sur carte du Natural Resources Intelligence Service. Le poste de Mistassini est situé dans le voisinage de la Rupert.

93. SPECK, Frank G., 1931. "Approximate distribution of Eskimo in 
the Labrador peninsula and on the coasts in the seventieth and eighteenth centuries, based upon early sources, and approximate recorded distribution of Montagnais-Naskapi groups as referred to at the same period." Figure dans le texte. in: Speck, "Montagnais-Naskapi bands and early Eskimo distribution in the Labrador peninsula," American Anthropologist, Oct-Dec. 1931.

93a. Speck, Frank G., 1931. "Approximate location, since about 1850, of local groups or bands of Montagnais-Naskapi and Eskimo." Figure dans le texte. Dans l'ouvrage précité de Speck.

94. Jenness, Diamond, 1932. "Approximate distribution of the Eastern Algonkian tribes in 1525 A.D." Figure dans le texte. In: Jenness, "The Indians of Canada". National Museum of Canada 1932, $446 \mathrm{p}$.

Le territoire attribué aux Indiens Mistassini est sujet à revision.

95. Norman, G.W.H., 1936. "Approximate North West boundary of Precambrian gneisses and relationship of folded and faulted belts." In: Norman, "The Northeast trend of late precambrian tectonic features in the Chibougamau district, Québec, "Trans. Royal Soc. Canada, Ser. 3, Sect. IV, Vol. 30, 1936.

96. Da vidson, Daniel Sutherland, 1937. "Snowshoes". Memoirs of the American Philosophical Society, vol 6, 207 p., Philadelphia, 1937. Renferme dans le texte de nombreuses cartes de distribution relatives aux différents types de raquettes. Plusieurs de ces cartes intéressent la région du lac Mistassini.

97. Canoe Route No 4. 1937. "Canœ trip from lake St. John to lake Mistassini. (238 miles). "2 p. polycopiées accompagnant une carte (bleu) de 7.89 milles au pouce. Natural resources Intelligence Service, Department of the Interior, Ottawa, 1937.

A 1 usage des excursionnistes.

98. Canoe Route No 8, 1937. "Canœ trip. Oskelaneo to lake Chibougamau and lake Mistassini. (208 miles. "5 p. polycopiées accompagnant une carte (bleu) de 7.89 milles au pouce. Natural Resources Intelligence Service, Department of the Interior, Ottawa, 1937.

A l'usage des excursionnistes.

99. Norman, G.W.H., 1938. "Map of part of the Chibougamau district showing deposits left by the receding ice-sheet and beaches and terraces of glacial lake Barlow-Ojibway." Carte hors-texte $(39 \mathrm{~cm}$. x $30 \mathrm{~cm}$.). In: Norman, "The last pleistocene ice-front in Chibougamau district, Quebec, "Trans. Royal Society Canada, Ser. 3, sect. IV, Vol 32, 1938.

100. Norman, G.W.H., 1939. "Map of Mistassini district showing the change in character of glacial deposits north-east of Mistassini lake." Figure dans le texte. In Norman, "The south-eastern limit of glacial lake Barlow-Ojibway in the Mistassini lake region, Quebec," Trans. Royal Soc. Canada, Ser. 3, sect. IV, Vol. 33, 1939.

101. Michelson, Truman, 1939. "Distribution and interrelations of the Cree and Montagnais-Naskapi dialects." Carte dans le texte. In: 
Michelson, "Linguistic classification of Cree and Montagnais-Naskapi dialects," Smithsonian Institution, Bureau of American Ethnology, Anthropological papers, No 8, Washington 1939.

102. Norman, G.W.H., 1940. "Sketch map showing geology of Mistassini lake region". Carte dans le texte. In: Norman, "Thrust faulting of Grenville gneisses north-westward against the Mistassini series of Mistassini Lake, Quebec." Journal of Geology, Vol. 48, July-Aug. 1940.

103. Beaver, 1940. "Fur trade posts of the Hudson's Bay Company, 1940." Extrait de Beaver, $38 \mathrm{~cm}$. x $27 \mathrm{~cm}$.

104. Ministère des Terres et Forêts, QuéBec, 1941. "Territoire d'Abitibi et de Mistassini, Ministère des Terres et Forêts, Québec 1941." 6 milles au pouce. Carte en deux feuillets préparés sous la direction de $\mathbf{M}$. Georges Coté, directeur, et Chs Savary, géographe, du Service des Arpentages.

Jusqu'à une date récente, c'était la carte publiée la plus importante couvrant le territoire de Mistassini.

105. Hydrographic Service, Ottawa, 1942. "Mistassini. Air navigation edition. Hydrographic map service,Department of Mines and Resources 1942. 8 miles to an inch."

Aussi nouvelles éditions corrigées, 1943, 1945.

106. Speck, Frank G., and Erseley, Loren C., 1942. "Chart showing distribution of Montagnais-Naskapi bands of the Lower St. Lawrence and Labrador peninsula with approximate location (in numbers) of family hunting and trapping districts." Carte dans le texte. In: Speck and Eiseley, "Montagnais-Naskapi bands and family hunting districts of the central and southeastern Labrador peninsula," Proc. Amer. philosophical Soc., Vol. 85, Jan. 1942.

107. Kindle, E.D., 1942. "Preliminary map 42-4A. Brock river, Abitibi and Mistassini territories, Quebec. Scale 1/253,440 or 1 inch to 4 miles". $29 \mathrm{~cm}$. large x $45.5 \mathrm{~cm}$. haut. Carte hors texte in: Kindle, "Brook river map-area, Abitibi and Mistassini territories, Quebec. Summary account." Dep. of Mines and Resources, Mines and Geology branch, Geological survey, Paper 42-4, 1942.

108. Burgesse, J.A., 1943. Montagnais cross-bow. The Beaver, outfit 274, p. 37-39, (Winnipeg) Dec. 1943. Renferme dans le texte carte de distribution de l'arbalette.

109. Lepage, abbé Ernest, et Dutilly, P. Arthème, 1945. Retracing the route of Michaux's Hudson's Bay journey of 1792. Revue de l'Université d'Ottawa, janvier-mars 1945. Renferme dans le texte une carte donnant l'itinéraire des auteurs de même qu'en insertion, la carte de Brunet (no 63, supra).

110. Ministère des Terres et Forêts, Québec, 1946. "Province de Québec. Département des Terres et Forêts... La ligne frontière Québec / Terre-Neuve n'est pas indiquée sur cette carte pour cause. Echelle: 16 milles 
au pouce. 1946. Georges Cote, directeur des arpentages. Chs F. Chartre, cartographe." 4 feuillets.

Le territoire de Mistassini se trouve sur le feuillet sud-ouest. Aussi réimpression des feuillets sud avec indication des districts judiciaires.

111. W $\mathrm{AHL}, \mathrm{W} . G ., 1947$. "Témiscamie river area, Mistassini territory, Quebec. Geology by W.G. Wahl. - Région de la rivière Témiscamie, Territoire de Mistassini, Québec. Géologie par W.G. Wahl." Carte hors texte, $58.5 \mathrm{~cm} . \times 47 \mathrm{~cm}$., accompagnant Wahl, "Rapport préliminaire sur la région de la rivière Témiscamie, territoire de Mistassini", Ministère des Mines (Québec), Service de la carte géologique, 1947. (Aussi édition anglaise).

112. Hudson's Bay Company. 1947. "Map of the Dominion of Canada showing the establishment of the Hudson's Bay Company. Fur trade department, Winnipeg Canada. Scale 1:6,336,000 or 100 miles to 1 inch. Published Aug. 1947." $90 \mathrm{~cm} . \times 62.5 \mathrm{~cm}$.

113. [ Delanglez, P. Jean, and Rousseau, Jacques ], 1948. "Route of Jolliet to Hudson Bay. Routes from Lake St. John to lake Mistassini." In: Delanglez, "Life and voyages of Louis Jolliet, (1645-1700), Institute of Jesuit History publications, Chicago, 1948.

Carte, dans le texte (p. 167) refaite sous la direction du Père Delanglez d'après les notes et cartes manuscrites de Jacques Rousseau.

114. Rousseau, Jacques, 1948. "Trajet présumé de Louis-Jolliet depuis la Tómiscamie jusqu'à la rivière à la Marte." Carte dans le texte, in Delanglez, op. cit. (voir no 113), p. 253.

115. Rousseau, Jacques, 1948. Le voyage d'André Michaux au lac Mistassini en 1792. Revue d'Histoire de l'Amérique française, 2 (No 3): 390-423. 1948. Aussi tirage à part, Mémoires du Jardin botanique de Montréal (réimprimé avec changement de pagination et quelques corrections), 34 p. 1948. Renferme les deux cartes suivantes, dans le texte: "Moyens d'accès au lac Mistassini"; "Trajet d'André Michaux de la rivière Mistassini au lac Mistassini."

La seconde carte, trop finement lettrée, a du être refaite pour le tirage à part des Mémoires du Jardin botanique de Montréal.

116. Neilson, James N., 1949. "Région du lac Albanel. Territoire de Mistassini. Carte préliminaire no 699”. $58 \mathrm{~cm}$. x $47 \mathrm{~cm}$. Carte hors texte accompagnant: Neilson, Rapport préliminaire sur la région du lac Albanel, Territoire de Mistassini. Ministère des Mines (Québec), Service de la carte géologique, R.P. No 224, 1949. 